\title{
GUEST EFFECT ON NEMATIC-ISOTROPIC PHASE TRANSITION TEMPERATURE OF LIQUID CRYSTAL 6CHBT
}

\author{
D. Bauman, J. Solczyński and E. Chrzumnicka \\ Institute of Physics, Poznań University of Technology \\ Piotrowo 3, 60-965 Poznań, Poland
}

(Received May 13, 1997; in final form August 1, 1997)

\begin{abstract}
The polarized absorption spectra of seven dichroic azo dyes dissolved in the nematogenic liquid crystal host 6 CHBT have been recorded as a function of temperature. On the basis of these spectra, the guest order parameter has been determined. Moreover, the influence of the dye addition on the behaviour of the guest-host mixture in the vicinity of the nematic-isotropic phase transition has been examined. The experimental results have been compared in some details with the calculations done on the basis of the mean field theory for binary mixtures.
\end{abstract}

PACS numbers: 61.30.Gd, 64.70.Md

\section{Introduction}

Liquid crystal displays (LCDs) are recently the fastest growing display technology because of such advantages as low power consumption, low voltage operating, flatness, easiness of fabrication, flexible design, low costs. The most common LCDs used today are based on the twisted nematic (TN) principle [1]. An alternative display technique consists in incorporating a dichroic dye (guest) in a liquid crystal matrix (host) and using the guest-host effect $[2,3]$. Guest-host liquid crystal displays (GH LCDs) can work with one, or even no polarizers [4] and therefore the viewing cone is very wide and uniform and the brightness is significantly higher than in the case of TN LCDs. However, in order to construct GH LCD possible to the practical application, a number of factors must be taken into account.

It had been previously found that a dichroic dye added to a liquid crystal affects the host order parameter [5-8] and moreover, the molecular orientation depends on the dye concentration $[6,7,9]$. It had been also observed that addition of a dichroic dye to a nematic host changes the nematic-isotropic phase transition temperature $\left(T_{\mathrm{NI}}\right)$ causing either an increase or a decrease in $T_{\mathrm{NI}}$ of the pure liquid crystal [6-14]. 
In this paper, a detailed study of the influence of some dichroic azo dyes on the nematic-isotropic phase transition temperature of liquid crystal 6CHBT 4-(trans-4'-n-hexylcyclohexyl)isothiocyanatobenzene was made. This liquid crystal has technological meaning, it is widely applied as a component of liquid crystalline mixtures used in LCDs [15]. An attempt to find a correlation between the molecular geometry of the guest, the order parameter and the clearing temperature of the azo dye-6CHBT mixtures was undertaken.

\section{Experimental}

Materials: Seven azo dyes of various molecular sizes and shapes were used as guest species. The molecular structure, the wavelength of absorption maximum in 6 CHBT, $\lambda_{\max }$, the molecular weight, $M$, and the ratio of molecular length to breadth, $l / d$, for the dyes investigated are gathered in Table I. $l / d$ values were estimated from the known bond lengths and molecular configurations [16], including van der Waals radii of the terminal atoms [17]. All the dyes were synthesized and chromatographically purified in the Institute of Dyes, Łódź University of Technology, Poland.

The nematogen liquid crystal 4-(trans-4'-n-hexylcyclohexyl)isothiocyanatobenzene was synthesized in the laboratory of Prof. R. Dąbrowski, Military Academy of Technology in Warsaw and used without further purification; the observed transition temperatures were in good agreement with those given in literature [18].

The dyes were initially screened for their solubility in 6CHBT in the nematic phase and were added to the liquid crystal at a concentration changed from $0.3 \%$ wt to either upper limit of the solubility or $3 \% \mathrm{wt}$.

Apparatus: The polarized absorption spectra of the dye-6CHBT mixtures were recorded in the visible spectral region by means of a SPECORD M40 spectrophotometer (Carl Zeiss Jena) equipped with polarizers. The measurements were made as a function of temperature using "sandwich" cells of a $10 \mu \mathrm{m}$ thickness. The temperature of the cells was regulated and controlled with a practical accuracy of $\pm 0.1^{\circ}$. After changing the measurement temperature, the cell was left for approximately $30 \mathrm{~min}$ to reach a thermal equilibrium. The homogeneous alignment of the liquid crystal and dye molecules was made by a treatment of the glass surfaces of the cells with polyimide and by rubbing. The molecular alignment in such a cell was parallel to the surfaces, and the quality of orientation was controlled with the aid of the crossed polarizers. The absorption spectra of the dyes dissolved in 6CHBT were recorded for two positions of the cell, that is, with the orientation axis of the liquid crystal sample at angles of $0^{\circ}$ and $90^{\circ}$ with respect to the polarization plane of the light beam. Additionally, the base line spectra for pure $6 \mathrm{CHBT}$ were recorded for the same two positions of the cell. After correction for the base line, two components of absorbance, $A_{\|}$and $A_{\perp}$, as a function of the wavelength were obtained.

The temperatures of the nematic-isotropic phase transition, both for pure $6 \mathrm{CHBT}$ and for dye-6CHBT mixtures, were determined with an accuracy of $\pm 0.1^{\circ}$ by means of a polarizing microscope equipped with a heating stage. 
TABLE I

Dyes used in experiment.

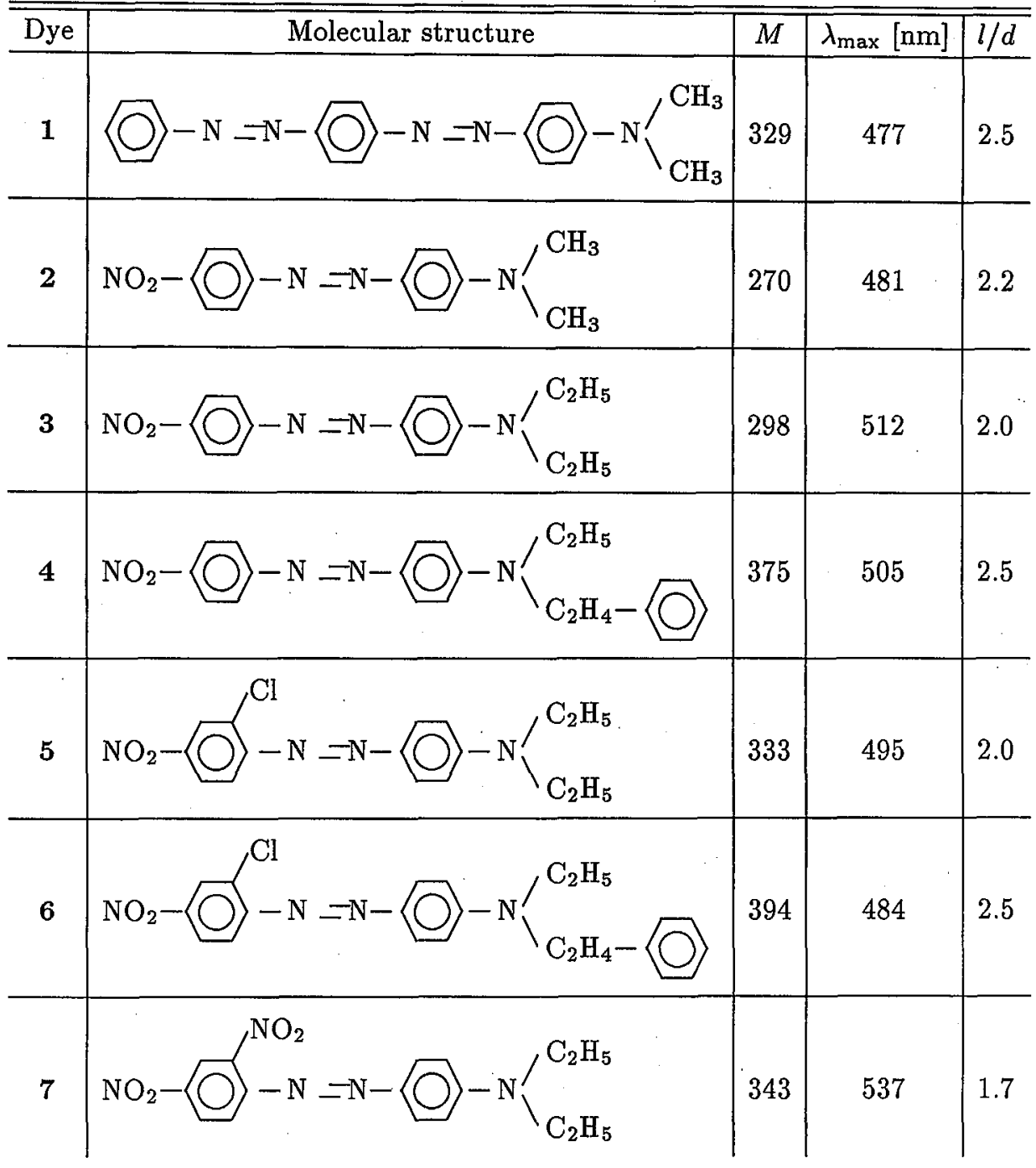

\section{Results and discussion}

\subsection{Order parameter of dye in liquid crystal matrix}

A characteristic feature of the nematic phase of liquid crystalline substance is the existence of the long range orientational order, which means that the elongated liquid crystal molecules tend to arrange parallel to each other and parallel to a certain preferred direction, described by the director, $n$. Owing to thermal motion, this direction is an average value in space or time. The measure of the efficiency of molecular orientation along the director is the order parameter, intro- 
duced by Zvetkoff [19] and defined through the Legendre polynomial of the second order, $\left\langle P_{2}\right\rangle$

$$
\left\langle P_{2}\right\rangle=S_{\mathrm{H}}=\frac{1}{2}\left\langle 3 \cos ^{2} \theta-1\right\rangle,
$$

where the angular bracket represents the time or space average, and $\theta$ is the angle between the long molecular axis of the liquid crystal host molecule and the director.

Equation (1) can be also applied to determine the order parameter of the guest dissolved in the liquid crystal matrix, but $\theta$ is now defined as an angle between the director and the long molecular axis of the guest. Experimentally, the order parameter of the dichroic dye, $S_{\mathrm{G}}$ can be estimated from the spectrophotometric data by applying the following equation $[20,21]$ :

$$
S_{\mathrm{G}}=\frac{A_{\|}-A_{\perp}}{A_{\|}+2 A_{\perp}} \frac{2}{3 \cos ^{2} \alpha-1}
$$

where $A_{\|}$and $A_{\perp}$ are the absorbances at $\lambda_{\max }$ for the dye in the host medium, when the light is polarized, respectively, parallel and perpendicular to the director, and $\alpha$ is the angle between the linear absorption transition dipole moment, $\mu$, and the long axis of the dye molecule.

In the case of the dyes, for which one can assume $\alpha=0^{\circ}$, the order parameter can be directly calculated from the equation given by White and Taylor [4]

$$
S_{\mathrm{G}}=\frac{A_{\|}-A_{\perp}}{A_{\|}+2 A_{\perp}} \text {. }
$$

Figure 1 shows the polarized components of the absorption spectrum, $A_{\|}$and $A_{\perp}$, for a $0.3 \%$ solution of dye 3 in 6 CHBT. Such absorption curves are typical of all azo dyes investigated - only one absorption band in the visible spectral region is observed. However, the position of $\lambda_{\max }$ depends strongly on the terminal and lateral substituents. The substituents affect also the order parameter, $S_{\mathrm{G}}$, which is

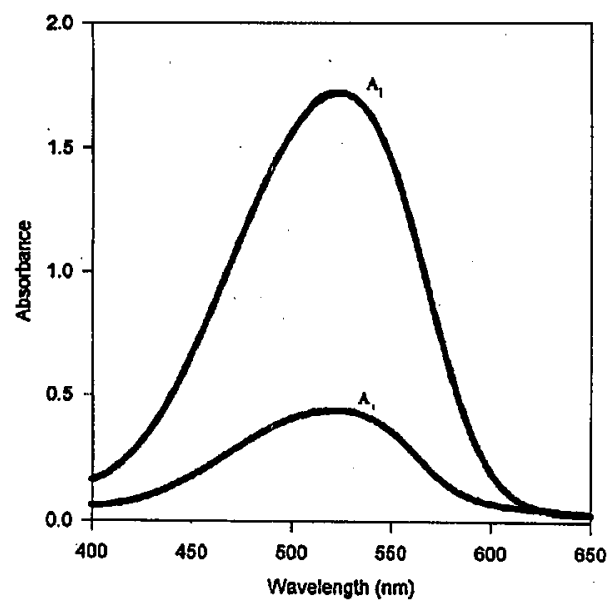

Fig. 1. Spectra of polarized components of absorption, $A_{\|}$and $A_{\perp}$, for dye 3 in 6 CHBT. 

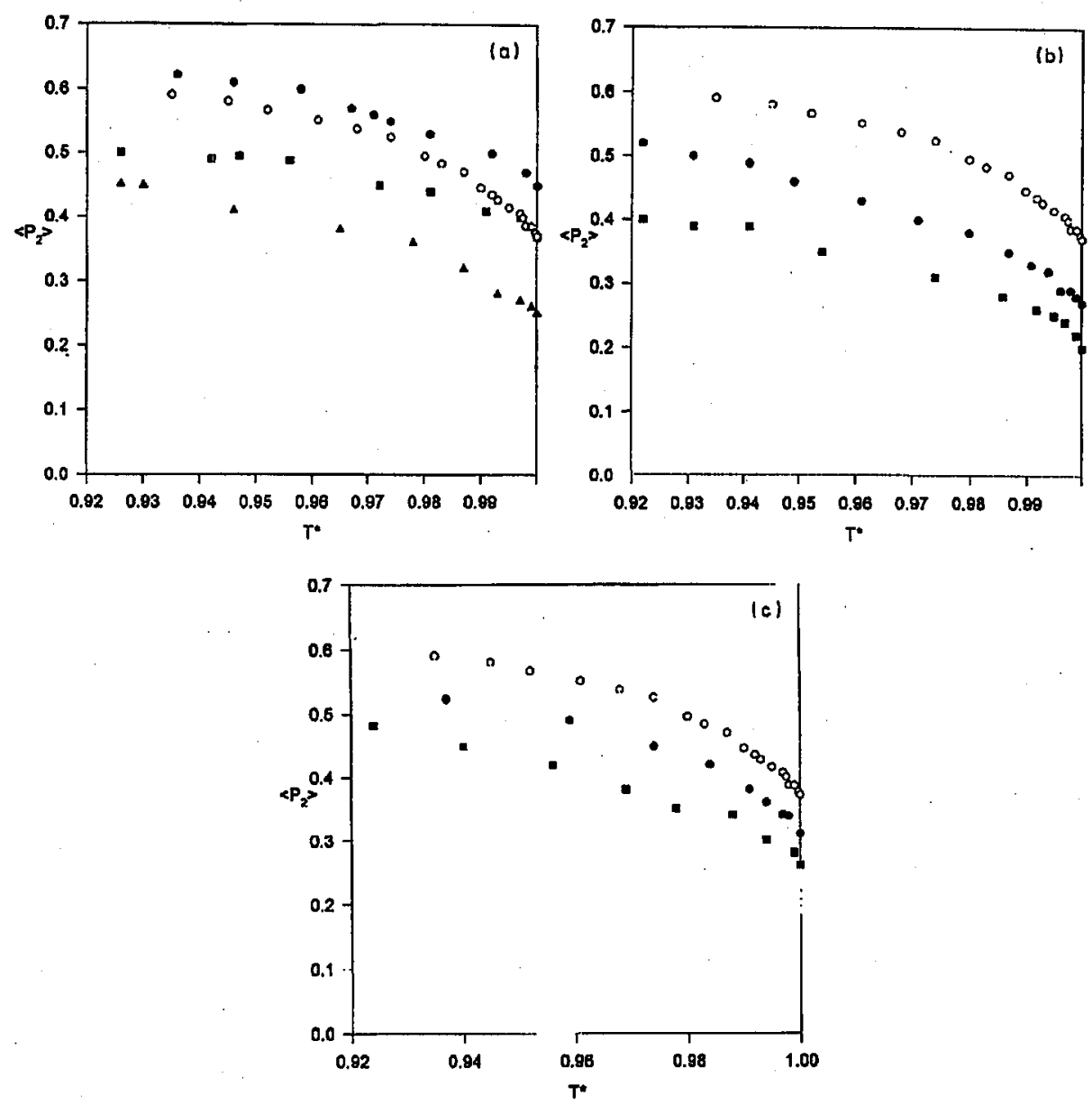

Fig. 2. Temperature dependence of order parameter (a) for dyes 1 (circles), 2 (squares) and 7 (triangles) in 6CHBT, (b) for dyes 3 (circles) and 4 (squares) in 6CHBT, (c) for dyes 5 (circles) and 6 (squares) in 6CHBT and $(a, b, c)$ for pure 6CHBT [22] (open circles).

illustrated in Figs. 2. These figures present the order parameter $S_{\mathrm{G}}$, estimated from the polarized absorption spectra and Eq. (3) for azo dyes dissolved in 6CHBT at a concentration of $0.3 \% \mathrm{wt}$ as a function of the reduced temperature $T^{*}=T / \bar{T}_{\mathrm{NI}}$, where $\bar{T}_{\mathrm{NI}}$ is the average clearing temperature of the guest-host mixture and $T$ is the temperature of the measurement in $\mathrm{K}$. Additionally, the temperature dependence of the host order parameter, $S_{\mathrm{H}}$ for pure 6CHBT, determined from the optical birefringence measurements [22] is plotted in the figures. At least three and usually five cells were used to obtain each order parameter, and the results presented here are average values. The uncertainty in the determination of $S_{\mathrm{G}}$ was estimated to be \pm 0.01 . 
TABLE II

Guest order parameter of azo dyes dissolved in 6CHBT.

\begin{tabular}{c|c|c}
\hline \multirow{2}{*}{ Substance } & \multicolumn{2}{|c}{$S$} \\
\cline { 2 - 3 } & $\begin{array}{c}\text { at room } \\
\text { temperature }\end{array}$ & $\begin{array}{c}\text { at } \mathrm{N} \rightarrow \mathrm{I} \\
\text { transition }\end{array}$ \\
\hline dye $\mathbf{1}$ in 6CHBT & 0.62 & 0.44 \\
dye $\mathbf{2}$ in 6CHBT & 0.50 & 0.37 \\
dye 3 in 6CHBT & 0.52 & 0.27 \\
dye 4 in 6CHBT & 0.40 & 0.20 \\
dye 5 in 6CHBT & 0.58 & 0.31 \\
dye 6 in 6CHBT & 0.48 & 0.26 \\
dye 7 in 6CHBT & 0.45 & 0.25 \\
pure 6CHBT & 0.58 & 0.37
\end{tabular}

Table II summarizes the values of the maximal guest order parameter found for the dyes investigated in $6 \mathrm{CHBT}$ at the room temperature $(300 \mathrm{~K})$ as well as $S_{\mathrm{G}}$ obtained in the vicinity of the nematic-isotropic phase transition. For comparison, $S_{\mathrm{H}}$ for $6 \mathrm{CHBT}$ at the same temperatures are given too.

From the results presented in Fig. 2 and in Table II it can be seen that the order parameter decreases with the rise of temperature and drops to the certain specified value at the nematic-isotropic transition. In all the cases this transition is of the first order and in the isotropic phase the value of $S$ is equal to zero. Only in the case of dye 1 the order parameter $S_{G}$ is greater than the order parameter of 6 CHBT at the same reduced temperature, which suggests that the dye with two azo-linkage can improve the molecular orientation in the guest-host mixture. The presence of the additional benzene ring in the end group of the dye causes the decrease in the order parameter (compare $S_{\mathrm{G}}$ for dyes $\mathbf{3}$ and $\mathbf{4}$ and dyes 5 and 6). This can be due to the non-zero angle between the long molecular axis and the transition moment, $\boldsymbol{\mu}$ in this case and/or to steric effects, which reduce the guest-host attractive interactions leading to the perturbation of the close alignment of the dye and liquid crystal molecules. The lateral $\mathrm{Cl}$ atom in the 3-position (dye 5 and 6) improves the efficiency of the molecular orientation. Such observations had been made also previously for other guest-host mixtures [12, 14, 23]. However, the presence of the nitro group (dye 7) in the 3-position leads to the decrease in the order parameter and it means that this group is sterical hindrance for the efficient dye molecular orientation in the liquid crystal medium. The temperature dependence of $S_{\mathrm{G}}$ as well as of the $S_{\mathrm{G}} / S_{\mathrm{H}}$ ratio is different for various azo dye-6CHBT mixtures, which leads to the conclusion that the mixtures investigated are differently resistant to thermal fluctuations (vibrations, librations and possible internal reorientations of the molecules) and indicates strong individual molecular interaction of azo compounds with the liquid crystal host. 


\subsection{Nematic-isotropic transition temperature of guest-host mixtures}

A dichroic dye added to a nematic liquid crystal influences not only the mesophase range of a nematic host, causing either a decrease or an increase in the clearing point, but leads to the formation of a two-phase region in the vicinity of the nematic-isotropic phase transition [7, 8, 12-14, 24]. The existence of such region is in accordance with the thermodynamic laws and the first-order nature of the phase transition for binary mixtures [25].

TABLE III

Thermal data for dye-6CHBT mixtures (concentration $2 \% \mathrm{wt}$ ).

\begin{tabular}{c|c|c|c|c|c|c}
\hline \hline Dye & $T_{\mathrm{I}}[\mathrm{K}]$ & $T_{\mathrm{N}}[\mathrm{K}]$ & $T_{\mathrm{I}}-T_{\mathrm{N}}$ & $\Delta T_{\mathrm{I}}$ & $\Delta T_{\mathrm{N}}$ & $\bar{T}_{\mathrm{NI}}$ \\
\hline $\mathbf{1}$ & 318.6 & 318.0 & 0.6 & +2.6 & +2.0 & 318.30 \\
$\mathbf{2}$ & 315.9 & 315.8 & 0.1 & -0.1 & -0.2 & 315.85 \\
$\mathbf{3}$ & 312.6 & 311.9 & 0.7 & -3.4 & -4.1 & 312.25 \\
$\mathbf{4}$ & $\mathbf{3 1 0 . 9}$ & 309.4 & 1.5 & -5.1 & -6.6 & 310.15 \\
$\mathbf{5}$ & 314.6 & 314.0 & 0.6 & -1.4 & -2.0 & 314.30 \\
$\mathbf{6}$ & 312.3 & 310.8 & 1.5 & -3.7 & -5.2 & 311.55 \\
$\mathbf{7}$ & 314.7 & 314.2 & 0.5 & -1.3 & -1.8 & 314.45
\end{tabular}

Table III and Fig. 3 gather the results of the thermal investigations of azo dye-6CHBT mixtures. Here $T_{\mathrm{I}}$ and $T_{\mathrm{N}}$ denote the temperatures, at which, on cooling, the two-phase region begins (the first nematic drop appears) and ends (the isotropic phase entirely disappears), respectively. $\left(T_{\mathrm{I}}-T_{\mathrm{N}}\right)$ is the range of the two-phase region and $\Delta T_{\mathrm{I}}$ and $\Delta T_{\mathrm{N}}$ are the shifts of $T_{\mathrm{I}}$ and $T_{\mathrm{N}}$ with respect to $T_{\mathrm{NI}}$ of the pure liquid crystal, whereas $\bar{T}_{\mathrm{NI}}$ denotes the average nematic-isotropic transition temperature for the mixtures. $T_{1}^{*}$ and $T_{N}^{*}$, given in Fig. 3, are defined as follows: $T_{\mathrm{I}}^{*}=T_{\mathrm{I}} / T_{\mathrm{NI}}$ and $T_{\mathrm{N}}^{*}=T_{\mathrm{N}} / T_{\mathrm{NI}}$, and $x$ is the dye mole fraction. $T_{\mathrm{NI}}$ for $6 \mathrm{CHBT}$ is equal to $316 \mathrm{~K}$.

The data presented in Table III and in Fig. 3 indicate that the changes of the clearing temperature of $6 \mathrm{CHBT}$ after the guest addition as well as the breadth of the two-phase region strongly depend on the molecular structure of the dye and on the dye concentration. Comparing the temperature data with the results in Table II, one can also see that there exists some correlation between the shift of $T_{\mathrm{NI}}$ in the presence of the dye and the relation of the guest order parameter with respect to the host order parameter. For dye 1, which has $S_{\mathrm{G}}>S_{\mathrm{H}}$, the shift of $T_{\mathrm{NI}}$ is positive, whereas for dyes 3-7 $\left(S_{\mathrm{G}}<S_{\mathrm{H}}\right)$ the clearing temperature of the guest-host mixtures is always lower than for pure 6CHBT. In the case of dye 2, for which $S_{\mathrm{G}} \simeq S_{\mathrm{H}}$ in the vicinity of the nematic-isotropic transition, the clearing temperature remains almost constant up to the concentration of $3 \% \mathrm{wt}$. The largest decrease in $T_{\mathrm{NI}}$ is observed for dyes 4 and 6 , which is probably connected with the existence of the heavy end group in the molecular structure of these dyes, causing the destruction of the orientation and the destabilization of the mesophase. For the mixtures of $6 \mathrm{CHBT}$ with these two dyes also the widest two-phase region occurs. 

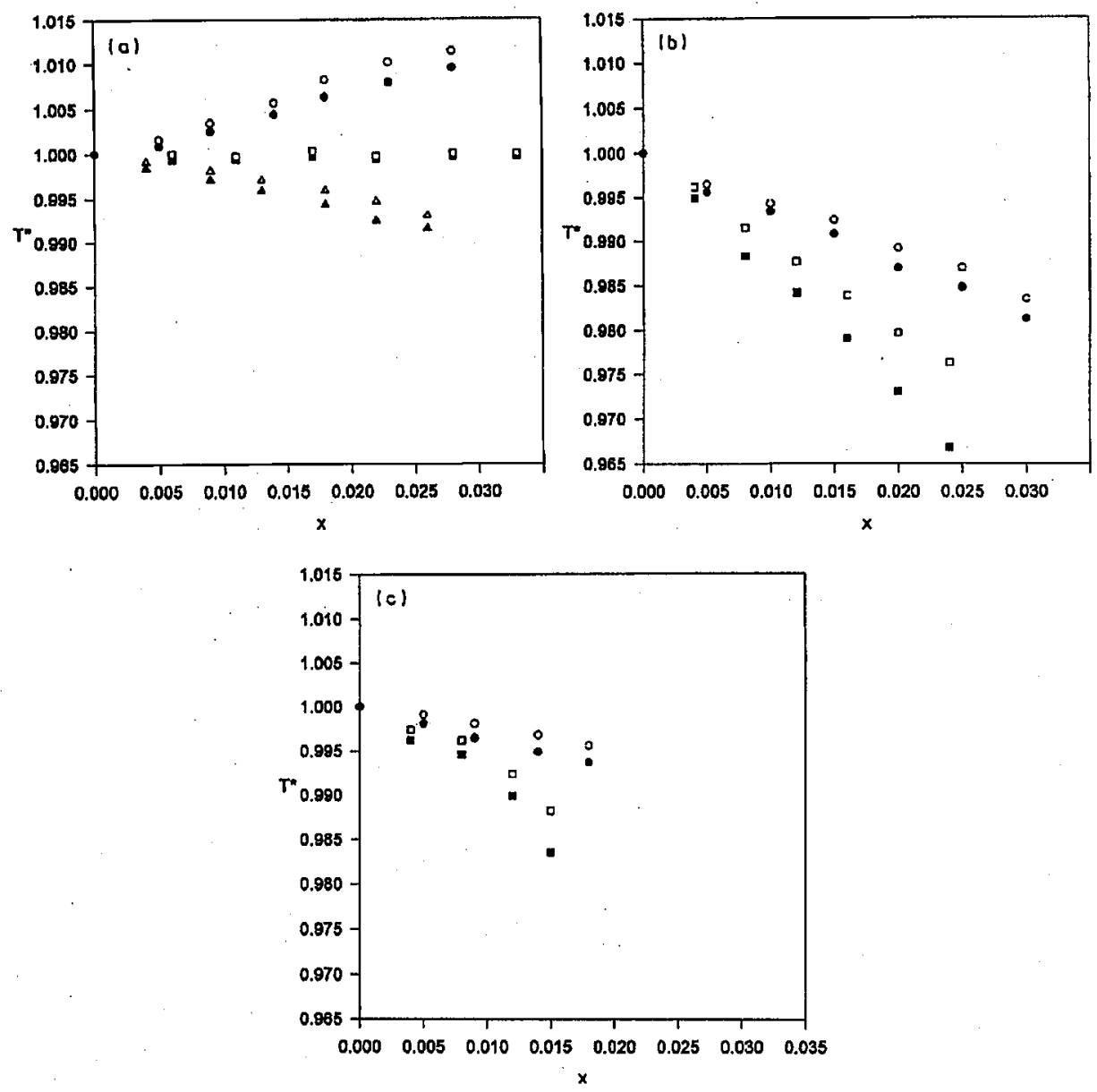

Fig. 3. Reduced temperature $\left(T^{*}\right)$ vs. dye mole fraction $(x)$ diagram (a) for dyes 1 (circles), 2 (squares) and 7 (triangles) in 6CHBT; (b) for dyes 3 (circles) and 4 (squares) in $6 \mathrm{CHBT}$; (c) for dyes 5 (circles) and 6 (squares) in $6 \mathrm{CHBT}, T_{\mathrm{I}}^{*}$ - open symbols, $T_{\mathrm{N}}^{*}$ - filled symbols.

\subsection{Comparison of experimental results with theoretical predictions}

The indicators of the order-destruction or the order-enhancement in the nematic phase can be the slopes of the changes of $T_{\mathrm{N}}^{*}$ and $T_{\mathrm{I}}^{*}$ lines with the change in the guest mole fraction, $x$, defined in the following way:

$$
\beta_{\mathrm{N}}=\frac{\mathrm{d} T_{\mathrm{N}}^{*}}{\mathrm{~d} x}, \quad \beta_{\mathrm{I}}=\frac{\mathrm{d} T_{\mathrm{I}}^{*}}{\mathrm{~d} x} .
$$

A positive $\beta$ means the ability of a guest to the stabilization, and negative $\beta$ to the destabilization of the nematic phase of the liquid crystalline host.

From thermodynamics it follows [25] that the limiting slopes $\left(x \rightarrow 0, T^{*} \rightarrow 0\right)$ of the nematic and isotropic boundary lines, $\beta_{\mathrm{N}}^{\infty}$ and $\beta_{\mathrm{I}}^{\infty}$, respectively, are given 
by

$$
\beta_{\mathrm{N}}^{\infty}=\left(1-\frac{\gamma_{\mathrm{N}}^{\infty}}{\gamma_{\mathrm{I}}^{\infty}}\right) \frac{R}{\Delta S_{\mathrm{NI}}}, \quad \text { and } \quad \beta_{\mathrm{I}}^{\infty}=\left(\frac{\gamma_{\mathrm{I}}^{\infty}}{\gamma_{\mathrm{N}}^{\infty}}-1\right) \frac{R}{\Delta S_{\mathrm{NI}}},
$$

where $R$ is the gas constant, $\Delta S_{\mathrm{NI}}$ is the nematic-isotropic transition entropy of the pure host, and $\gamma_{N}^{\infty}$ and $\gamma_{I}^{\infty}$ are the infinite dilution (Henry's law) guest activity coefficients in the nematic and isotropic phases, respectively.

In order to determine the ratio $\gamma_{N}^{\infty} / \gamma_{I}^{\infty}$, the experimentally obtained $\beta$ values have been corrected to the infinite dilution using the procedure described by Kronberg et al. [26]. The $\Delta S_{\mathrm{NI}} / \mathrm{R}$ value for $6 \mathrm{CHBT}$ has been assumed to be 0.267 , following [13]. The corrected $\beta_{N}^{\infty}$ and $\beta_{\mathrm{I}}^{\infty}$ slopes as well as the ratios $\gamma_{\mathrm{N}}^{\infty} / \gamma_{\mathrm{I}}^{\infty}$ for azo dyes dissolved in 6CHBT are listed in Table IV.

TABLE IV

Comparison of experimental values of $\beta_{\mathbb{N}}^{\infty}, \beta_{\mathrm{I}}^{\infty}$ and $\gamma_{N}^{\infty} / \gamma_{\mathrm{I}}^{\infty}$ with theoretical calculations for dye-6CHBT mixtures.

\begin{tabular}{|c|c|c|c|c|c|c|c|c|c|}
\hline \multirow{3}{*}{ Dye } & \multirow{2}{*}{\multicolumn{3}{|c|}{ Experiment }} & \multicolumn{6}{|c|}{ Theory } \\
\hline & & & & \multicolumn{3}{|c|}{$l / d=3.1$} & \multicolumn{3}{|c|}{$l / d=2.2$} \\
\hline & $\beta_{\mathrm{N}}^{\infty}$ & $\beta_{\mathrm{I}}^{\infty}$ & $\gamma_{\mathrm{N}}^{\infty} / \gamma_{\mathrm{I}}^{\infty}$ & $\beta_{\mathrm{N}}^{\infty}$ & $\beta_{1}^{\infty}$ & $\gamma_{\mathrm{N}}^{\infty} / \gamma_{\mathrm{I}}^{\infty}$ & $\beta_{\mathrm{N}}^{\infty}$ & $\beta_{\mathrm{I}}^{\infty}$ & $\gamma_{N}^{\infty} / \gamma_{I}^{\infty}$ \\
\hline 1 & +0.34 & +0.42 & 0.89 & +0.55 & +0.66 & 0.85 & +0.27 & +0.28 & 0.93 \\
\hline 2 & 0.00 & +0.01 & 1.00 & +0.26 & +0.28 & 0.93 & -0.04 & -0.04 & 1.01 \\
\hline $\mathbf{3}$ & -0.63 & -0.54 & 1.16 & -0.12 & -0.11 & 1.03 & -0.41 & -0.37 & 1.11 \\
\hline 4 & -1.33 & -0.99 & 1.36 & -0.56 & -0.45 & 1.14 & -0.94 & -0.74 & 1.25 \\
\hline 5 & -0.36 & -0.23 & 1.08 & +0.04 & +0.04 & 0.99 & -0.24 & -0.21 & 1.06 \\
\hline 6 & -0.98 & -0.71 & 1.23 & -0.33 & -0.30 & 1.09 & -0.70 & -0.60 & 1.19 \\
\hline 7 & -0.33 & -0.24 & 1.08 & -0.12 & -0.11 & 1.03 & -0.34 & -0.30 & 1.09 \\
\hline
\end{tabular}

A smaller ratio $\gamma_{N}^{\infty} / \gamma_{1}^{\infty}$ implies greater compatibility with the nematic phase of the host and vice versa, thus correlation between the activity coefficients and the ordering of the guest by the host would be anticipated. Comparing the results from Table IV with those from Table II one can see that dye 1 which shows $S_{\mathrm{G}}>S_{\mathrm{H}}$ has $\gamma_{\mathbb{N}}^{\infty} / \gamma_{1}^{\infty}<1$, thus its molecular axes are able to align parallel to the long axes of the liquid crystal molecules. This dye stabilizes the nematic host causing the broadening of the mesophase region. However, for case where $S_{\mathrm{G}}<S_{\mathrm{H}}$ the dye added to the liquid crystal depresses the clearing temperature, leading to the destruction of the host mesophase.

According to the model based on the mean-field theory of nematics $[27,28]$ and the extension proposed by Humphries et al. for binary mixtures [29] the expression for the ratio of the infinite-dilution activity coefficients in the vicinity of the clearing point is following:

$$
\ln \left(\frac{\gamma_{\mathrm{N}}^{\infty}}{\gamma_{\mathrm{I}}^{\infty}}\right)=\frac{\Delta S_{\mathrm{NI}}}{R}\left[\frac{\nu_{2}}{\nu_{1}}-\left(\frac{b_{12}}{b_{11}}\right)^{2}\right]
$$

where $\nu_{1}$ and $\nu_{2}$ are, respectively, the host and guest molecular volumes, while ratio $b_{12} / b_{11}$ is a measure of the relative strength of the guest-host and host-host 
molecules interactions. The $b_{12} / b_{11}$ ratio can be easily estimated from the measurements of the $S_{\mathrm{G}} / S_{\mathrm{H}}$ in the vicinity of the nematic-isotropic transition [29]. Knowing the $\gamma_{N}^{\infty} / \gamma_{\mathrm{I}}^{\infty}$ values, the slopes $\beta_{\mathrm{N}}^{\infty}$ and $\beta_{\mathrm{I}}^{\infty}$ can be calculated from Eqs. (4a) and $(4 \mathrm{~b})$, respectively.

Similarly as in the previous papers $[7,8,13,14]$, the ratio of the molecular length to breadth was used as a measure of the host and guest sizes. It was assumed that $\nu_{1} / \nu_{2}=m_{1} / m_{2}$, where $m=l / d$. The value of $l / d$ for the monomeric molecules of 6CHBT has been estimated similarly to those for the dyes molecules (see Table I) and is equal to $3: 1$.

The calculated values of $\beta_{\mathrm{N}}^{\infty}, \beta_{\mathrm{I}}^{\infty}$ and $\gamma_{\mathrm{N}}^{\infty} / \gamma_{\mathrm{I}}^{\infty}$ for azo dyes in 6CHBT are gathered in Table IV. The comparison of the data presented in this table reveals the fact that in some cases the correlation between experimental and calculated results is not satisfactory, both for $\beta^{\infty}$ and $\gamma_{\mathbb{N}}^{\infty} / \gamma_{I}^{\infty}$ values. Thus, in the next fitting on, the flexibility of the alkyl chain of the liquid crystal molecules has been taken into account $[30,31]$ and $l / d$ value for $6 \mathrm{CHBT}$ has been assigned as 2.2 . The results are presented also in Table IV. In this case the much better correlation between calculations and experiment has been achieved, which demonstrates that the theoretical model which takes into account the flexibility of the host end chain can describe well the behaviour of the guest-host mixtures at the nematic-isotropic phase transition.

Finally, it is worth noting that the data obtained from the theoretical predictions confirm both the existence of the two-phase region observed experimentally in the vicinity of the clearing point as well as the exact correlation between the ability of the alignment of the elongated guest molecules parallel to the long molecular axes of the host and the nematic-isotropic transition temperature for the dye-liquid crystal mixtures.

\section{Acknowledgments}

This work was financially supported by the Poznań University of Technology Research Project No. DS 62-133/5.

\section{References}

[1] M. Schadt, W. Helfrich, Appl. Phys. Lett. 18, 127 (1971).

[2] G.H. Heilmeier, L.A. Zanoni, Appl. Phys. Lett. 13, 91 (1968).

[3] G.H. Heilmeier, J.A. Castellano, L.A. Zanoni, Mol. Cryst. Liq. Cryst. 8, 293 (1969).

[4] D.L. White, G.N. Taylor, J. Appl. Phys. 45, 4718 (1974).

[5] J. Altschuh, R. Weiland, J.V. Kosack, H.-G. Kuball, Ber. Bunsenges. Phys. Chem. 88, 562 (1984).

[6] M. Kozielski, D. Bauman, M. Drozdowski, Z. Salamon, Mol. Cryst. Liq. Cryst. 142, 1 (1987).

[7] D. Bauman, Mol. Cryst. Liq. Cryst. 159, 197 (1988).

[8] D. Bauman, Mol. Cryst. Liq. Cryst. 174, 1 (1989).

[9] W. Haase, O. Trinquet, U. Quotschalla, J.K. Foitzik, Mol. Cryst. Liq. Cryst. 148, 15 (1987). 
[10] F.C. Saunders, L. Wright, M.G. Clark, in: Liquid Crystals and Ordered Fluids, Eds. A.G. Griffith, J.F. Johnson, Vol. 4, Plenum Press, New York 1984, p. 831.

[11] P. Diot, J.K. Foitzik, W. Haase, Phys. Rev. Appl. 20, 121 (1985).

[12] D. Bauman, H. Moryson, J. Jadżyn, Acta Phys. Pol. A 85, 553 (1994).

[13] D. Bauman, T. Martyński, E. Mykowska, Liq. Cryst. 18, 607 (1995).

[14] D. Bauman, A. Wąsik, J. Jadżyn, Liq. Cryst. 20, 195 (1996).

[15] R. Dąbrowski, Mol. Cryst. Liq. Cryst. 191, 17 (1990).

[16] Tables of Interatomic Distances and Configuration in Molecules and Ions, The Chemical Society, London 1958.

[17] A. Bondi, J. Phys. Chem. 68, 441 (1964).

[18] R. Dąbrowski, J. Dziaduszek, T. Szczuciński, Mol. Cryst. Liq. Cryst. 124, 241 (1985).

[19] V. Zvetkoff, Acta Phys. USSR 10, 557 (1939).

[20] R.W. Filas, M.M. Labes, J. Appl. Phys. 52, 3949 (1981).

[21] A.V. Ivashchenko, O.S. Petrova, V.V. Titov, Mol. Cryst. Liq. Cryst. 108, 51 (1984).

[22] G. Czechowski, B. Żywucki, D. Bauman, J. Jadżyn, in: Liquid Crystals: Materials Science and Applications, Eds. J. Żmija, Z. Raszewski, J. Zieliński, Proc. SPIE 2372, 163 (1995).

[23] D. Bauman, H. Moryson, J. Mol. Struct. 404, 113 (1997).

[24] D. Bauman, Z.-X. Fan, W. Haase, Acta Phys. Pol. A 80, 545 (1991).

[25] D.E. Martire, in: The Molecular Physics of Liquid Crystals, Eds. G.R. Luckhurst, G.W. Gray, Academic Press, London 1979, Ch. 10.

[26] B. Kronberg, D. Patterson, J. Chem. Soc. Faraday Trans. II 72, 1673 (1976).

[27] W. Maier, A. Saupe, Z. Natforsch. A 14, 882 (1959); ibid. 15, 287 (1960).

[28] R.L. Humphries, P.G. James, G.R. Luckhurst, J. Chem. Soc. Faraday Trans. II 68, 1031 (1972).

[29] Ref. [25], Ch. 11.

[30] H. Seki, T. Uchida, Y. Shibata, Mol. Cryst. Liq. Cryst. 138, 367 (1986).

[31] A.S. Paranjpe, K.U. Deniz, P.S. Parvathanathan, V. Arnithalingen, K.V. Muralidharan, Mol. Cryst. Liq. Cryst. 149, 79 (1987). 